\title{
La muerte clínica: un diagnóstico y un testimonio
}

\section{Clinical death: A diagnosis and a testimony}

\author{
J. L. Trueba
}

\section{RESUMEN}

La muerte clínica de una persona es la muerte del encéfalo. Hay un consenso bastante generalizado en nuestra medicina occidental sobre los criterios necesarios y el modo de proceder para un correcto diagnóstico de "muerte encefálica". En un caso concreto, cuando existen antecedentes razonables de daño cerebral catastrófico y determinados signos clínicos en la exploración neurológica, un médico con suficiente experiencia puede llegar a emitir un diagnóstico de muerte clínica.

Aceptando que el diagnóstico de muerte es un ejercicio de puro juicio práctico, y que como tal nunca puede dar certidumbre absoluta, debemos asumir que a pesar de la incertidumbre debemos tomar decisiones razonables y prudentes.

Sin embargo, existen opiniones discrepantes que consideran sería necesario un más alto grado de certe$\mathrm{za}$ antes de tomar decisiones.

Para tomar decisiones responsables se requiere intentar comprender el concepto de la muerte cerebral como un auténtico constructo cultural en el que son necesarios conocimientos a cerca de "qué" es lo que se define científicamente como muerte; el "cómo" se puede diagnosticar en la práctica; el "cuándo" se dice que un hombre está clínicamente muerto, y de "cual" es el grado de evidencia que conlleva el diagnóstico.

Palabras clave. Muerte encefálica. Muerte clínica. Diagnóstico. Ética.

An. Sist. Sanit. Navar. 2007; 30 (Supl. 3): 57-70.

\begin{abstract}
The clinical death of a person is the death of the brain. There is a fairly general consensus in western medicine on the necessary criteria and the mode of proceeding for a correct diagnosis of "brain death". In a specific case, with a reasonable previous record of catastrophic brain damage and certain neurological exploratory signs, an expert practitioner is able to diagnose clinical death.

Accepting that the diagnosis of death is an exercise of diagnostic judgement, in practical application, which cannot as such provide absolute certainty; we have to accept that, in spite of this uncertainty, we have to take sensible and prudent decisions.

However, there are dissenting opinions that believe in the need for a higher degree of certainty before taking decisions

It is necessary to try to understand the concept of "brain death" in order to be able to take responsible decisions. This concept is a real "cultural construction", in which it is necessary to have knowledge about the scientific definition of death, how death can be diagnosed in practice, when it is possible to say that someone is clinically dead, and what is the degree of evidence carried by the diagnosis.
\end{abstract} Ethics.

Key words. Brain death. Clinical death. Diagnosis.
Prof. Asociado de Neurología. Hospital Universitario Doce de octubre UCM.

Presidente de la Asociación de Bioética Fundamental y Clínica.

\section{Correspondencia:}

Juan Luis Trueba Gutiérrez

C/ López de Hoyos, 7, 5izq

28006 Madrid

Tfno. 915617816 


\section{LA MUERTE CLÍNICA: ALGUNAS CONSIDERACIONES TERMINOLÓGICAS}

La última palabra del final de la vida es la muerte. El final de la vida y la muerte son términos muy serios con los que los médicos afrontamos al problema de la existencia de una persona como viviente. Cuando de lo que se trata es del final de la vida de un hombre concreto nos estamos refiriendo al periodo final del proyecto vital de una persona y es aquí donde la palabra "muerte" adquiere su mayor trascendencia y complejidad.

El propósito de este trabajo es realizar una serie de consideraciones sobre los aspectos clínicos y los problemas éticos que suscita el diagnóstico de muerte al final de la vida de un hombre concreto. Este es el sentido de lo que llamaremos "muerte clínica", es decir: la actualidad presencial de la realidad de la muerte en quien yace, postrado clínicamente ${ }^{1}$, ante un médico, que tiene que afrontarlo, verificando y atestiguando mediante un juicio diagnóstico el "estado de muerte irreversible"; lo que permitirá tomar las decisiones pertinentes al respecto.

Al final de la vida humana, lo que en otro capítulo de esta monografía se aborda como "el morir", se refiere a terminalidad, pero no a la realidad de "la muerte"; las decisiones que se pueden y deben tomar en una u otra situación son muy distintas, siempre importantes, pero no igualmente trascendentes.

Conviene aclarar que desde un punto de vista conceptual no es lo mismo el término "el morir" que el de "muerte clínica", ya que el primero se refiere al proceso biológico del morir mientras que el segundo se refiere al momento en el que se diagnostica y atestigua un "nuevo estado"; el de estar muerto. Veamos las diferencias entre ambos términos.
En primer lugar, "el morir". Se refiere a un proceso asistencial de un paciente, con un pronóstico de situación evolutiva terminal. Es, pues, un pronóstico clínico dentro de una referencia lineal del tiempo de la vida humana; conlleva una predicción pronóstica de muerte próxima, lo que permite la consideración de que el proceso clínico está en estado terminal. El estado terminal plantea otros problemas: especialmente la futilidad de las decisiones terapéuticas y la posibilidad de una "limitación del esfuerzo terapéutico"'. Así, pues, "el morir", se refiere a la vida humana concreta como proceso biológico, y al pronóstico de terminalidad de la vida temporal biológica del sujeto; implica un pronóstico vital negativo, pero no afecta necesariamente la dimensión ontológica del sujeto personal, en tanto que su individualidad como todavía viviente no se cuestiona.

En segundo lugar, "la muerte". Puede referirse a un estado o a un evento. La muerte como estado es el opuesto a la vida; la muerte como evento es el fin de la vida, opuesto al nacimiento. El evento de la muerte es la culminación de la vida en un organismo vivo, mientras que el estado es lo que sucede a dicho evento ${ }^{3}$. Dicho en términos médicos: el evento equivale al juicio diagnóstico de muerte clínica; el estado al pronóstico que se deriva del diagnóstico; el estar muerto sin posibilidad de retorno a la vida, y en una situación que ha de conducir necesariamente a la desestructuración orgánica total del cuerpo: la putrefacción del cadáver en un tiempo inmediato. La "muerte clínica" al reconocer un "nuevo estado", compromete la dimensión ontológica del sujeto objeto de estudio; es un hecho real muy trascendente, ya que abre la posibilidad de actuaciones muy trascendentes como pueden ser: la firma de un parte de defunción, la retirada de la respiración asistida, o la solicitud de donación de órganos a la familia del fallecido.

1. Del griego $\chi \lambda \iota \nu \eta$ (chliné), lecho.

2. Trueba JL. Los marcos asistenciales de la limitación del esfuerzo terapéutico. En: J De la Torre (ed) La limitación del esfuerzo terapéutico. (Dilemas éticos de la medicina actual 20) Universidad Pontificia de Comillas, Salamanca 2006.

3. Definición de la muerte en la enciclopedia libre Wikipedia. 


\section{LA MUERTE CLÍNICA COMO JUICIO DIAGNÓSTICO: NECESIDAD DE DEFINIR EL MOMENTO DE LA MUERTE}

Si la muerte clínica es un evento diagnóstico que abre a la actualidad de un nuevo estado (la presencia de una realidad reconocible como muerte en quien yace ante el médico), será necesario definir de la manera más precisa posible los criterios que permiten reconocerla; los datos y hechos que son necesarios para admitir la irreversibilidad vital del organismo y la mera consideración del cuerpo como "cadáver".

¿En que momento tiene lugar eso que llamamos "muerte"? Esta es la cruz del asunto. En esencia ¿qué es la muerte? El problema del momento de la muerte tiene serias implicaciones a nivel práctico, y es un aspecto de gran interés desde el punto de vista judicial, filosófico, teológico y moral ${ }^{4-6}$.

Plantearse la pregunta de en qué momento tiene lugar lo que llamamos "muerte clínica" remite necesariamente a las preguntas esenciales sobre la "muerte humana". Ambas cuestiones no son caprichosas, pues, siendo el hombre el único animal que conoce la existencia de la muerte, y el único que conoce que todos necesariamente tenemos que morir, afrontar la muerte del "otro" y asumir la ineludible muerte propia, implican necesariamente a cada individuo como persona, y a todas las estructuras sociales en la reflexión y participación de la toma de decisiones, que no pueden quedar reducidas a un planteamiento exclusivamente científico o clínico asistencial.
Y es que no es fácil establecer una definición de muerte que pueda ser entendida y aceptada por todos. Sin embargo, la muerte clínica por tratarse de un problema práctico, esencialmente basado en hechos de experiencia, siempre será un problema ético que obligará a tomar decisiones desde la incertidumbre. No puede ser de otra manera ante un conocimiento empírico. No obstante, estamos obligados a tratar de perfeccionar nuestro conocimiento del proceso del morir, perfeccionar nuestros criterios y buscar cada vez criterios más sólidos para el diagnóstico de la muerte

Establecer criterios para definir lo mejor posible el momento de la muerte resulta ser una imperiosa necesidad fruto de un doble imperativo: ético y legal.

\section{EL IMPERATIVO ÉTICO EN EL DIAGNÓSTICO DE LA "MUERTE CLÍNICA"}

En trabajos anteriores me referí al problema que plantea desde un punto de vista práctico el hecho de que la muerte clínica ha de establecerse como un momento de un proceso evolutivo biológico y que ello supone necesariamente asumir la experiencia de que dicho momento no corresponde a un instante meramente temporal, sino que encierra un necesario momento de imprecisión y de incertidumbre: primero, incertidumbre temporal en cuanto al "donde" y "cuando"; segundo, incertidumbre existencial sobre que es "la muerte propia”, y tercero , incertidumbre esencial sobre que es la "muerte en sí".

El análisis histórico muestra la experiencia acumulada de que los criterios de muerte clínica han cambiado con los tiem-

4. D. W. Brock The role of the public in public policy on the definition of detah. En: Youngner SJ, Arnoold RM, SchapIRo R., eds. The definition of death: contemporary controversies. Baltimore: Johns Hopkins University Press, 1999: 293-307.

5. Pontificiae Academiae Scientiarum Scripta varia 60, pp. 113-114: Working Group on the Artificial Prolongation of Life and Dtermination of the exact moment of death, (C. CHAGAS, ed.), Cittá del vaticano 19-21 octubre 1985.

6. Pontificiae Academiae Scientiarum Scripta varia 83 Working Group sobre Determinación de la muerte cerebral y su relación con al muerte humana, 10-14 diciembre 1989 Edit. R.J. WHITE, H. AgstWurm y I CARRASCo DE PAULA.

7. Trueba, J.L. La muerte clínica. En: Diez palabras sobre el final de la vida. Edit: J. Elizari. Verbo divino 2006 
pos, y especialmente a partir del desarrollo tecnológico de la medicina.

Hoy en día es el médico el que diagnóstica la muerte; no siempre ha sido así. Determinar la muerte ha sido un acto de gran trascendencia para cualquier sociedad mínimamente organizada. El hecho de designar a un individuo como cadáver tiene consecuencias inmediatas: como, por ejemplo, la inhumación o la aplicación de lo que socialmente se denominan los "ritos de la muerte". Cada sociedad ha tenido los suyos propios y cada sociedad ha interpretado a su manera el sentido de la muerte y el modo de determinarla, explicarla y comprenderla. Los miedos con respecto a la posibilidad de un error en el diagnóstico de muerte y la experiencia de que en algunos casos, especialmente coincidiendo con los enterramientos masivos de guerras y epidemias, se han producido enterramientos con vida ${ }^{8}$, condujo por presión social a la implantación de leyes para los enterramientos, a los periodos de espera antes de proceder a la inhumación y especialmente a la necesidad de una certificación médica de la muerte. Hasta finales del siglo XVIII y principios del XIX la figura del médico estaba ausente de los ritos de muerte en las sociedades del mundo occidental. El médico acompañaba al paciente mientras "había algo que hacer", pero a partir de dicho momento procedía al "desahucio" del paciente que quedaba al cuidado de familiares, y de los miembros sociales y religiosos especialmente dedicados a las obligaciones morales y a los mandamientos de "enterrar a los muertos". El diagnóstico propiamente dicho de "muerte clínica" basado en la intervención de un médico para emitir un certificado legal "para" poder enterrar no se implantó hasta principios del siglo XIX, ligado al nacimiento de los criterios científicos de la medici- na moderna, y a razones de salubridad pública. La gestión de la muerte humana pasó de ser un patrimonio de la religión y la filosofía a ser un problema de "diagnóstico clínico", y la "muerte clínica" una cuestión sobre como actuar médicamente ante la evidencia de una pérdida irreversible de las funciones y las estructuras constitutivas del organismo de un viviente humano concreto.

Con todo esto el papel que la sociedad ha venido demandando al médico se ha ido complicando y elevando los niveles de responsabilidad exigida. Partiendo de unas obligaciones puramente asistenciales en la esfera del diagnóstico nosológico, la determinación del pronóstico y de las posibilidades terapéuticas sobre el cura, cuidar y consolar a los pacientes, el médico pasa a convertirse en garante de la existencia personal con unas obligaciones testimoniales de la mayor trascendencia. Los familiares y la sociedad misma espera de la actuación del médico con respecto al diagnóstico un testigo fiel de la misma; alguien que de fidelidad y testimonio de la muerte de una persona. En definitiva, no un mero diagnóstico incidental o procesual, sino lo que me atrevería a llamar "un diagnóstico testimonial de muerte clínica". Es aquí donde la rigurosidad del juicio diagnóstico ponderado razonable y prudente adquiere todo su valor. El médico que testifica el diagnostico de muerte clínica no es un mero evaluador, sino un testigo veraz, capaz de dar fe pública de quién y cuándo está muerto ${ }^{9}$.

El diagnóstico correcto de "muerte clínica" se realiza teniendo en cuenta una serie de signos objetivos que poseen valor diagnóstico mostrativo de "muerte". Estos signos no aparecen de repente al modo como los antiguos imaginaban la llegada de la muerte, como si fuese un personaje

8. Centanaro G.. El concepto de muerte. Un poco de historia. (http://www.geocities.com/ HotSprings/Spa/3516/muerceb.html?200527) refiere que Bruchier (1742) recogió 189 casos de supuestos enterramientos con vida.

9. Conviene distinguir entre evaluar y testimoniar. El diagnóstico de la muerte clínica es el de muerte como testimonio; un testimonio veraz no de hechos físicos, sino de acontecimientos humanos. Por ello, es un testimonio y no una mera comprobación. El médico atestigua la muerte; verifica la muerte, no simplemente la comprueba, porque atestación es algo infinitamente más que comprobación, cuando se trata de acontecimientos humanos. 
que aparece con su aspecto cadavérico y su guadaña. Tampoco la muerte ocurre porque ese personaje que nos visita nos conduzca a la muerte en un proceso danzante al que no podemos resistirnos. Son todas interpretaciones míticas que hoy casi parecen ridículas, pero que han tenido desde siempre una fuerza seductora frente a los enigmas que la muerte como final de la vida nos plantea.

Es interesante constatar como en dichas imágenes míticas se encuentra escondido no sólo el enigma sobre "cuando" aparece la muerte, sino también el enigma sobre cual es la "causa" de la muerte. Creo que ambos enigmas requieren un abordaje por separado que nos ayude a comprenderlos, pero no será vano dejar bien sentado que la muerte clínica no puede tener un sentido, al menos desde una posición a la altura de lo que la ciencias positivas nos han venido demostrado, sobre la base de hacer de ella una "cosificación" ni una relación subjetivista de lo que sin duda es una experiencia de hecho necesaria. En definitiva que no existe la "señorita muerte" como ente sustancial ni como "entidad morbosa"; la muerte no es ni una "persona" ni una "enfermedad" en su sentido más tradicional. Para el médico la muerte no es una entidad nosológica, sino un "estado" biológico; entra más en la categoría de síndrome que en el de entidad morbosa o enfermedad. Es, pues, un mero diagnóstico clínico de "estado", lo cual plantea como enseguida veremos cuestiones importantes sobre el "como" y el "cuando".

Quizá sea oportuno empezar abordando primero el problema médico del "como", esto es: la causa del posible "estado" de muerte. La razón es que todo diagnóstico clínico de muerte empieza por un afrontamiento ante un paciente concreto de la posibilidad de dicho estado. De si, en definitiva, el paciente está o no está muerto. Es una duda que requiere confirmación resolutoria, pero que aparece ante una situación concreta en la que siempre existen unos antecedentes conocidos, mediatos o inmediatos, que hacen plausible el razonamiento puramente lógico de que el planteamiento de la cuestión es oportuno. Es decir, resulta ante esa situación concre- ta razonable pensar en el estado de muerte, porque sencillamente existen "causas" suficientes para ello. Es el dilema médico de la "causa inmediata" de la muerte y de la "enfermedad previa" que figuran en los certificados médicos. Y hay que reconocer que frecuentemente los médicos, a la hora de certificar la muerte, tienen tendencia a pasar de puntillas sobre los temas que abordan las causas de la muerte, a pesar de ser tan trascendentes para la vida del paciente y las implicaciones sociales que conllevan. Ello no quiere decir que la causa suficiente no sea evidente para el clínico que evalúa el estado del paciente; no hay nadie que piense en la posibilidad de la "llegada de la muerte" de un paciente sin tener antes la evidencia de una causa concreta en la historia clínica que hace temer la existencia de un daño orgánico suficiente como para producir la muerte del organismo entero.

Las causas de la muerte pueden ser clasificadas de una manera práctica en dos grandes grupos fisiopatogénicos: muertes repentinas o inesperadas, y muertes terminales o esperables en el proceso final de la vida. En las primeras es posible reconocer un antecedente inmediato agudo que ha podido dañar de manera muy importante algún órgano vital o esencial para la vida (por ejemplo el cerebro o el corazón). En las segundas existe el antecedente de la "enfermedad previa" con una evolución pronóstica mala que hace esperable su desenlace como situación puramente terminal. En ambas se plantea la situación como razonablemente crítica para la vida, hasta el punto de considerar la posibilidad de la muerte. Es desde esta situación concreta de la que parte el juicio diagnóstico médico para establecer y confirmar el estado de muerte del paciente por daño irreversible estructural del organismo. Es decir el diagnóstico testimonial de una muerte clínica.

La desestructuración del organismo y la muerte celular no ocurren en el mismo instante en todas y cada una de las células que forman parte de un organismo entero; el ritmo de instauración y progresión es diferente para cada tejido y para cada órgano, y los llamados "signos de muerte" tienden a aparecer en una sucesión tempo- 
ral en la que sólo un juicio diagnóstico sobre los datos semiológicos conduce al médico a descubrir la evidencia de la muerte clínica de su paciente. La muerte clínica no se puede certificar por la existencia de un solo signo indicativo de muerte, aunque evidentemente hay algunos signos que tienen mayor valor que otros. En general son los signos que muestran la existencia de putrefacción de los tejidos los que fueron buscados por la medicina forense a la hora de determinar si un cadáver debía ser o no inhumado. Sin embargo son los signos llamados vitales la ausencia irreversible de latido cardiaco y de respiración los que el clínico ha venido considerando como causas inmediatas de la muerte de un paciente.

\section{LA MUERTE CLÍNICA POR PARADA CARDIACA Y RESPIRATORIA}

La ausencia de signos vitales y especialmente el criterio de la parada cardiorrespiratoria fue el modo tradicional de hacer el diagnóstico de muerte en la medicina. Su verificación era relativamente fácil, especialmente cuando la medicina científica aportó procedimientos más exactos para registrar el latido cardiaco y la respiración. Su confirmación final también era fácil ya que bastaba con esperar la aparición de signos de putrefacción para tener evidencias suficientes que evitasen los diagnósticos precipitados o erróneos de la muerte.

El fin último del diagnóstico de muerte por ausencia de signos vitales era "para" poder enterrar, y las razones del enterramiento respondían a preceptos religiosos y más tarde cuando en nuestras sociedades las normas de salubridad pública se establecieron como una necesidad social, a preceptos legales perfectamente establecidos.

Las maniobras de reanimación cardiopulmonar supusieron un gran impacto sobre las creencias establecidas de la hora de la muerte. En efecto, la posibilidad de que una pérdida de funciones vitales como la respiración y el latido cardiaco fuesen reversibles, y que determinadas maniobras permitiesen un "retorno a la vida", planteó una cuestión muy seria sobre los criterios reales de una muerte definitiva.

En definitiva, la parada cardiorrespiratoria es un criterio sólido de muerte clínica solamente cuando existe "voluntad de no reanimar" lo que frecuentemente también supone una estrategia de "limitación del esfuerzo terapéutico" (LET).

Así, pues, la muerte por parada cardiorrespiratoria es el primer modo de realizar el diagnóstico de muerte clínica en el final de la vida. El "momento de la parada" es considerado como "el momento de la muerte", y a partir de ese evento, el médico puede firmar el certificado de defunción ya que desde el punto de vista legal la sociedad civil considera que la vida propiamente humana del paciente ha terminado; que el cuerpo esta en estado cadavérico y que puede ser enterrado después de transcurridas las 24 horas preceptivas.

La parada irreversible de la función cardiaca y respiratoria supone la muerte del organismo como consecuencia de la anoxia de los órganos y tejidos corporales que en tiempo muy corto conduce a la muerte de las células, la destrucción por lisis de los órganos y la apertura al proceso de desintegración o descomposición del cadáver. Pero como la muerte celular no ocurre al mismo tiempo en todos los tejidos, y el órgano más sensible a la anoxia es el cerebro, la parada cardiorrespiratoria sin voluntad de reanimación produce en unos pocos minutos la muerte total del encéfalo; conlleva la ineludible e irrecuperable pérdida de las funciones de todo el encéfalo por lisis neuronal en cuestión de minutos. En definitiva, la muerte clínica real solo ocurre con la muerte de todo el encéfalo, como enseguida veremos, aunque en muchos casos dicha muerte encefálica haya estado precedida de una parada funcional cardiorrespiratoria irreversible o sin voluntad de reanimación.

En conclusión, la pérdida irreversible de las funciones llamadas "vitales" (latido cardiaco y respiración) ha sido el criterio diagnóstico tradicional para certificar la "muerte clínica" y la actuación del médico tenía una justificación basada fundamentalmente en motivos medicole- 
gales, y con la finalidad última de poder enterrar; era un diagnóstico "para poder enterrar", y el enterramiento una necesidad social preceptiva por razones de salubridad pública.

\section{LA MUERTE ENCEFÁLICA UN NUEVO "CONSTRUCTO CULTURAL" PARA LA MUERTE CLÍNICA}

Con el desarrollo tecnológico de la medicina y especialmente con la creación de las unidades de cuidados intensivos se abrieron nuevos caminos y modos de realizar los diagnósticos de la "muerte clínica".

Cuando los pacientes ingresados en UVI en estado de coma y con asistencia ventilatoria no salían de dicha situación clínica, se comenzaron a plantear nuevas dificultades para diagnosticar la muerte. A partir de 1956 comenzó a hablarse de casos de "coma irreversible" y de las incertidumbres que sobre el modo correcto de proceder planteaban. Así, por ejemplo: tomar decisiones sobre si retirar o no la asistencia ventilatoria; reanimar o no las paradas cardiacas; mantener o limitar el esfuerzo terapéutico; iniciar o no técnicas extraordinarias como la diálisis, etc. Pero también una cuestión ética fundamental de carácter ontológico: si los pacientes se mantenían en ausencia completa de función neurológica, sin retirar la intubación, y manteniendo el soporte cardiocirculatorio ise estaba manteniendo una vida humana o practicando "encarnizamiento terapéutico" y futilidad sobre un cadáver? La necesidad de tomar decisiones para afrontar estos graves problemas llevó a la búsqueda de un consenso social en los campos de la medicina, la ciencia, el derecho y la religión.
En 1968, el Comité de la Facultad de Medicina de Harvard ${ }^{10}$ constituido por diez médicos, un abogado, un teólogo y un historiador formula el primer criterio para la determinación de la muerte basado en un total y permanente daño encefálico, acuñándose el concepto de "muerte cerebral" o encefálica ${ }^{11}$. Desde entonces han aparecido numerosas revisiones sobre "muerte encefálica" que han consolidado el uso del término y en especial la Comisión del Presidente de Estados Unidos que en 1981 estableció el "Estatuto de muerte" en los siguientes términos: "Un individuo en el que se mantiene un cese irreversible de todas las funciones del encéfalo, incluyendo el tallo cerebral, está muerto" ${ }^{12-14}$. A partir de este momento la definición de la muerte clínica como "el cese permanente de todas las funciones vitales" quedaría circunscrita y aceptada médicamente al "cese permanente de la función del organismo como un todo" y teniendo en cuenta que el "encéfalo como un todo" es el responsable de la función del organismo como un todo, la muerte encefálica es equivalente a muerte clínica.

En resumen, podemos concluir que en nuestras sociedades del mundo occidental existen en el momento actual dos criterios válidos para llegar al diagnóstico clínico de muerte, que son:

Criterio cardiopulmonar: la comprobación del cese irreversible de la función cardiopulmonar (ausencia de latido cardiaco y respiración).

Criterio encefálico: la comprobación del cese irreversible de la función del encéfalo como un "todo" (no necesariamente de todas las neuronas), aún en presencia de un funcionamiento cardiovascular y ventilatorio artificial.

10. "Harvard Criteria". Report of Ad Hoc Committee of Harvard Medical School to Examine the Definition of Brain Death. A definition of irreversible coma. JAMA 1968; 205: 337-340.

11. En español es más correcto utilizar el término "muerte encefálica" ya que el término cerebro se utiliza de manera restringida a la porción encefálica constituida solo por los hemisferios cerebrales.

12. Medical Consultants to the President's Comisión. Guidelines for Determination of Death JAMA 1981; 246: 2184-2186.

13. Guidelines for Determination of Death. JAMA 11/13/1981; 246(19): 2184-2186.

14. Practice parameters for determining brain death in adults (summary statement). Neurology 1995; 45: 1012-1014. 
Es importante aclarar que no existen dos clases de muerte ni dos formas diferentes de morir, sino simplemente dos modos de llegar al diagnóstico de la muerte clínica y que esto es sólo una consecuencia de la necesidad de tener que tomar decisiones éticas ante la incertidumbre clínica de determinadas situaciones producidas por la medicina tecnológica y los medios artificiales de soporte cardiopulmonar. Ya he dicho que en definitiva la muerte clínica por criterio cardiopulmonar fisiopatogénicamente no es más que un modo de muerte encefálica aguda como consecuencia de la anoxia cerebral. En definitiva la muerte clínica de una persona es la muerte del encéfalo. Y que el concepto de "muerte encefálica" solamente surge cuando el paciente está con un control asistido de sus funciones cardiovasculares, es decir cuando está en una unidad de "cuidados intensivos" y conectado a un respirador.

\section{NECESIDAD DE ESTABLECER UNOS PARÁMETROS PRÁCTICOS PARA LA DETERMINACIÓN DE LA MUERTE ENCEFÁLICA}

De cuanto llevamos dicho se puede extraer una consecuencia: en presencia de medios artificiales de soporte cardiopulmonar la muerte clínica debe ser determinada por pruebas de función encefálica lo que abre la necesidad de establecer unos criterios diagnósticos precisos y rigurosos para el diagnóstico de muerte encefálica, ya que las consecuencias que dicho diagnóstico conlleva son serias y trascendentes.

Hay un consenso bastante generalizado en nuestra medicina occidental sobre los criterios necesarios y el modo de proceder para un correcto diagnóstico de "muerte encefálica". No es mi intención exponer en este lugar el protocolo de actuación para establecer el diagnóstico de muerte encefálica ni los problemas que encierra su aplicación ya que los he abordado en otros lugares ${ }^{15,16}$.

Existen numerosas revisiones sobre el tema ${ }^{17-19}$, diferentes propuestas de protocolo para establecer el diagnóstico y distintas normativas legales en los diversos países, sobre todo para establecer la muerte encefálica en los casos de donación de órganos ${ }^{20}$. Es también un hecho que existen opiniones divergentes sobre los mismos problemas, todo lo cual pone de manifiesto que el debate aunque con suficiente consenso no deja de estar abierto ${ }^{21-23}$. El debate se ha reactivado en los últimos años, especialmente centrado en los aspectos neurológicos sobre la especificidad y selectividad del concepto "muerte

15. TRUEBA J L La muerte cerebral como evidencia clínica. En: Bioética un diálogo plural. Homenaje a Javier Gafo Fernandez, S.J. J. FERRER y J. MARTINÉz (eds). Universidad pontificia de Comillas, 2002; 201-220.

16. TRueba J.L. ¿Qué implicaciones éticas tiene el diagnóstico de muerte encefálica? Retirada de las medidas de soporte y donación de órganos Medicina Intensiva vol 24 ํㅡㄹ 2 Febrero 2000.

17. WIJDICKS EFM. Brain death wordwide: Accepted fact but no global consensus in diagnostic criteria. Neurology 2002; 58: 2025.

18. WiJdicks EFM. The diagnosis of Brain Death. N Engl J Med, vol 344, $\mathrm{n}^{\circ} 16$ april 19, 20011215-1221

19. Henneman, EA, KARRAS GE 2004 Determining Brain Death in Adults: A Guideline for Use in Critical Care. Crit Care Nurse 24: 50-56.

20. Canadian criteria. Guidelines for diagnosis of brain death. Canadian Neurocritical Care Group. Can J Neurol Sci 1999; 26: 64-66.

21. VAN NoRman GA, A Incorrect application of accepted criteria. matter of life and deth. Anestesiology 1999; 91: 275-287.

22. DoIG CJ AND BuRGeSS E. Brain Death: resolving inconsistences in the ethical declaration of death. Can J Anesth 2003; 50(7): 725-731.

23. Rosenbaum, S. Ethical conflicts. Anesthesiology 1999;91:3-4. "Si hay algún tema en la legislación sanitaria y en la bioética del que se puede decir que esta bien resuelto, y a la vez persistentemente no resuelto, es el de determinar que ha tenido lugar la muerte". 
encefálica" y sus problemas de aplicación en la práctica ${ }^{2431}$.

En mi opinión, tal debate traduce las dificultades de aceptar el hecho de que el diagnóstico de la muerte es un ejercicio de un juicio razonable dentro de una aplicación práctica que nunca puede dar certeza, lo que nos obliga no solo a establecer criterios claros y rigurosos que siempre son potencialmente mejorables, sino, lo que me parece más importante, a asumir que no por su incertidumbre necesaria podemos dejar de tomar resoluciones y a justificarlas desde lo meramente razonable y prudente. Nadie estaría dispuesto a tolerar la actitud meramente pasiva ante la posibilidad suficientemente fundada de muerte; el horror a la putrefacción ante nuestros ojos de los seres queridos parece que ha gozado siempre del respaldo social por considerarlo un imperativo ético de primer orden que responde al mandato preceptivo de enterrar a los muertos. Así se ha considerado siempre, y aunque las condiciones físicas en las que se encuentran los que yacen en un lecho han cambiado mucho el reto tecnológico no puede llevarnos al absurdo de querer tener certezas absolutas donde nunca podrá haberlas. No se puede ir más lejos; de ahí la importancia de tales juicios diagnósticos que me he atrevido a catalogar de testimoniales. Diego Gracia nos lo ha dicho de manera insuperable; a sus propias palabras me remito.

"Las discusiones sobre el momento de la muerte y sobre el comienzo de la vida se hallan con frecuencia viciadas por esta obsesión de determinar con exactitud el momento preciso en el que la vida comienza o termina. Mi opinión es que todo eso carece de sentido. No digo que no haya que poner un razonable cuidado en la determinación del momento del inicio y del momento del final de la vida. Pero ni es posible conocer esos momentos con exactitud matemática, ni tiene sentido plantear así las cosas. La tesis que quiero defender es que hay que plantear estos problemas en una dirección completamente distinta. Entre el nacimiento y la muerte lo que se extiende es la vida. Este es el tema, la vida, no el momento de su comienzo y de su fin. Hay un tiempo de morir, porque el argumento de la vida se ha plenificado ya" ${ }^{\prime 2}$.

Planteadas las cosas así, el diagnóstico de "muerte clínica", hecho por un médico ante un paciente que se encuentra presente corporalmente ante él, postrado en un lecho y con antecedentes causales suficientes y determinados signos exploratorios neurológicos es una evidencia suficiente y razonables como para considerarlo "cadáver". Esto es, muerto por pérdida irreversible de todas sus funciones encefálicas. Si ello ocurre sin tener soportes vitales acce-

24. StePhen T. MERnoff, MD. Clinical Assistant profesor of Neurology, Brown Medical School. Brain Death: the Neurologist's Perspective.

http://www.brown.edu/Departments/Center_for_Biomedical_Ethics/ppt.

25. Truog, R.D. Brain Death-Too Flawed to Endure, Too Ingrained to Abandon (Demasiadas inperfecciones para durar y demasido asumidas para abandonarlas) $2007 \mathrm{~J}$ Laww Med Ethics 35: 273-281.

26. Bratton SL, Kolovos, NS, Roach, ES, Mcbride, V, Geiger, JL, Meyers, Rl 2006. Pediatric Organ Transplantation Needs: Organ Donation Best practices. Arch Pediatr Adolesc med 160: 468-472.

27. Shaner, DM, OrR, RD, Drought, T, Miller, RB, Siegel, M . Really, most Sincerely dead: Policy and procedure in the diagnosis of death by neurologic criteria. Neurology 2004. 62:1683-1686.

28. DoIG, CJ, BURGESS, E Brain deth: resolving inconsistencies in the ethical declaration of death. Canadian J. Anesthesia 2003; 50: 725-731

29. SwASh, M, BERESFORD, R, 2002. Brain detah: Still-unresolved issues worldwide. Neurology 58: 9-10.

30. Cranston, RE, Rockoff, MA, Thompson, JE, Larson, MD, Gray, AT, Truog, RD, Robinson, WM, Broyde, MJ, WIJDICKS, EFM, CAPRON, AM 2001. The diagnosis of Brain Death. NEJM 345: 616-618.

31. CAPron, AM 2001 Brain Death. Well Settled yet Still Unresolved. NEJM 344: 1244-1246.

32. D. Gracia Salir de la vida. En: Como arqueros al blanco. Estudios de bioética. Edit. Triacastela (San Sebastián) 2004, cap. 18, pp 395-431. 
sorios el paciente estará en parada cardiaca y respiratoria y la aparición de signos de putrefacción es inminente en horas. Si, por el contrario, el paciente está con soporte ventilatorio, y este no se retira, podría durar en esta situación algunos días hasta que finalmente se produzca la parada cardiaca definitiva. En ambos casos, la muerte clínica adviene por "muerte encefálica" y para el clínico su valoración diagnóstica es idéntica; sencillamente considera que se encuentra ante "un cadáver", y que puede ser razonable y prudente firmar el certificado de defunción una vez que el cadáver haya sido desentubado puesto que habría indicación de "limitación del esfuerzo terapéutico".

Como puede verse se trata de un diagnóstico clínico hecho por un médico; una actuación transitiva del médico que se basa en criterios objetivos de la exploración neurológica y que se encuentran incluidos entre las notas o características de lo que la ciencia describe como formando parte de la realidad mortal biológica humana. Es por lo tanto ante la ciencia y para el médico la realidad de la "muerte biológica" a cuyo conocimiento llega por experiencia práctica, pero fundamentada en unos hechos sobre los cuales ha de asentar su "juicio diagnóstico". El juicio diagnóstico abre la posibilidad de argumentar las razones por las que el estado del paciente lo valora como "cadáver".

\section{NECESIDAD DEL JUICIO DIAGNÓSTICO TESTIMONIAL EN EL CONCEPTO DE MUERTE CLÍNICA}

Me he detenido deliberadamente en la descripción de la situación diagnóstica de la "muerte clínica" para resaltar que en definitiva se trata de un juicio clínico que solo puede argumentarse desde lo razonable y prudente, apoyada en la búsqueda y valoración de los hechos más objetivos, de los procedimientos de ratificación diagnóstica más sofisticados y perfectos, pero que no puede, por tratarse de datos de experiencia práctica, dar certeza absoluta sobre el juicio emitido.

Es posible que alguien piense que esto es algo poco exacto para un tema tan serio. A estos únicamente podríamos res- ponder argumentando que "así es la vida, y así es la muerte". Así es, y así probablemente lo seguirá siendo, pero, aún con su incertidumbre, nos obliga como "realidad" que es a afrontarla, y a tomar resoluciones con respecto a ella. No podemos evitar el compromiso y la necesidad de actuar según sea nuestro juicio y nuestra valoración circunstancial. Todo proceso orgánico o biológico encierra un componente de incertidumbre que nos obliga a descubrir y asumir cierta dosis de relativismo científico y práctico para nuestras tomas de decisión. Esto puede resultar incomodo pero no es posible establecer un criterio absolutamente absoluto para poder certificar la muerte, pero ello no invalida la posibilidad de un diagnóstico testimonial, razonable y responsable.

En la práctica el concepto de muerte cerebral se genera desde un trágico dilema como consecuencia del desarrollo tecnológico de la medicina. El conocimiento y aplicación de las medidas de soporte cardiopulmonar, las unidades de cuidados intensivos y los medicamentos vasoactivos o los antibióticos han permitido mantener con vida situaciones que habrían muerto en épocas no demasiado remotas, pero también conducen a situaciones de daños irreversibles y a prolongar innecesariamente la llegada de la muerte, en lo que se ha denominado "encarnizamiento terapéutico". Por otra parte el desarrollo de las técnicas de trasplante de órganos vitales, como el corazón, hígado o pulmones y el desarrollo de tratamientos eficaces para impedir el rechazo inmunológico han venido planteando necesidades crecientes de donación de órganos. De una parte hay la urgente necesidad de encontrar órganos disponibles para trasplantar a pacientes enfermos que de otra manera morirían. Pero por otro lado es concebible que para que un paciente escape de una muerte inminente y cierta, lo debe hacer gracias una "muerte" de la que no se puede tener absoluta certeza.

Con todo para tomar decisiones responsables se requiere intentar comprender el concepto de la "muerte cerebral" como un auténtico "constructo" cultural en el que son necesarios conocimientos a cerca de qué es lo que se define científica- 
mente como muerte; el "como" se puede diagnosticar en la práctica; el "cuando" se dice que un hombre está clínicamente muerto, y de "cual" es el grado de evidencia o certidumbre conlleva el diagnóstico. También es necesario conocer cuales son las posibles decisiones que el diagnóstico de muerte cerebral permite plantear como opciones, y procurar facilitar el cumplimiento y fomento de las voluntades anticipadas como medio para establecer los niveles asistenciales que cada cual prefiera cuando deba pasar el trance de asumir la muerte propia.

\section{LAS PREGUNTAS FUNDAMENTALES PARA UN JUICIO DIAGNÓSTICO TESTIMONIAL DE LA MUERTE CLÍNICA}

Intentaré abordar sucintamente algunas de las cuestiones que en otros escritos he considerado esenciales para la realización de un juicio diagnóstico neurológico de la muerte encefálica.

1. ¿Qué es la muerte encefálica?

Es un concepto que emana del desarrollo propio y del momento empírico de la ciencia médica actual. Un fruto intelectual o "constructo cultural" que requiere para su comprensión conocimientos científicos racionales objetivamente justificados.

En general, dentro de lo que denominamos cultura occidental, los médicos, los trabajadores de la sanidad, los miembros pertenecientes a las iglesias de confesión cristiana, y los pertenecientes al mundo de las leyes, aceptan hoy en día plenamente "el estatuto de muerte": que un individuo en el que existe un cese irreversible de todas las funciones del encéfalo, incluyendo el troncoencéfalo, está muerto. En ausencia de soporte cardiopulmonar la muerte debe ser determinada por la ausencia prolongada de signos vitales (diagnóstico de muerte por el criterio cardiopulmonar: pérdida de la función espontánea cardiaca y pulmonar). En presencia de medios de soporte cardiopulmonar, la muerte debe ser determinada por pruebas de función encefálica (diagnóstico de muerte por el criterio encefálico: cese irreversible de la función del encéfalo como un "todo"). En ambas circunstancias la determinación de muerte debe ser realizada de acuerdo con los estándares médicos aceptados" ${ }^{33}$.

A partir de este momento, el concepto nuevo requiere una definición. La definición actual que es la que generalmente se acepta en nuestra cultura occidental ha sido formulada de la siguiente manera: se entiende por muerte cerebral encefálica, o neurológica, la pérdida irreversible de la función del cerebro y el troncoencéfalo ${ }^{34}$.

Desde aquí se han levantado algunas críticas a la misma, así como diversos intentos de modificarla o de diferenciar distintos subtipos de muerte neurológica ${ }^{35}$ atendiendo a razones anatómicas y estructurales de las lesiones observadas ${ }^{36,37}$.

Tres aspectos de la definición que merecen interés:

a) La muerte cerebral no es un concepto que se defina por criterios estructurales o anatomopatológicos, sino por criterios de función o de posibilidades funcionales del encéfalo.

b) La pérdida irreversible de esas funciones debe ocurrir en todo el encé-

33. Acta sobre la determinación de la muerte". President's Comision of the Study of Ethical Problems in Medicine and Biochemical Research. Defining death: a report on the medical, legal and ethical issues in the detrmination of death. Washington, D.C.: Government Printing Office, 1981.

34. Practice parameters for determinig brain death in adults (summary statement). report of Quality Standars Subcommittee of American Academy of Neurology. Neurology, 1995; 45: 1012-1014.

35. Escalante J.L. La definición de muerte. En: Trasplantes de órganos: problemas técnicos, éticos y legales. J. GAFO (de.) Universidad Pontificia de Comillas, 1996.

36. Henneman, E. A., KarRas, G.E. JR. Determining Brain Death in Adults: A Guideline for Use in Critical Care. Crt Care Nurse 2004; 24: 50-56.

37. Morenski, J.D., Oro, J.J., Tobias, J.D., Singh, A. Detrmination of Death by Neurological Criteria. J. Intensive Care Med 2003; 18: 211-221. 
falo (tanto en las funciones reconocibles del cerebro como en las correspondientes al troncoencéfalo).

c) La definición parece enfocar el punto de vista desde un planteamiento holístico, entendido como "un todo funcional", con soporte estructural encefálico, pero sin especificar cuáles son las funciones imprescindibles que definirían el encéfalo como "un todo".

\section{2. ¿Por qué es muerte cerebral?}

Porque cumple unos criterios que permiten un juicio racional diagnóstico que soporta la confrontación con la definición actual o vigente de muerte cerebral ${ }^{38}$.

En efecto, los criterios actuales recogidos en las directrices ("guidelines") de la Academia Americana de Neurología (AAN) establecen los llamados criterios prácticos para el diagnóstico clínico de muerte cerebral $^{39}$.

En el caso de España, la Organización Nacional de Trasplantes (ONT), y por ello, el Real Decreto ${ }^{40}$ que regula en la legislación española los trasplantes recoge en su anexo I un "Protocolo de diagnóstico y certificación de la muerte para la extracción de órganos de donantes fallecidos" que especifica de manera muy similar los criterios necesarios para el diagnóstico ${ }^{41-45}$.

Los criterios diagnósticos recogidos a partir de la AAN y resumidos en el Anexo I del R.D. 2070/199946, establecen, que la evidencia diagnóstica se alcanza con un proceso de valoración, no existiendo dato, signo, criterio o prueba diagnóstica que de una forma aislada, y por sí misma, permita hacer el diagnóstico de muerte cerebral. Los criterios son todos necesarios pero no suficientes para el diagnóstico, que requiere la síntesis, con un juicio racional, o de valoración ponderada, de los datos objetivos. La síntesis conduce a la evidencia emergente según la propia experiencia del clínico que valora.

Es comprensible, por lo tanto, que los criterios para el diagnóstico de muerte cerebral recogidos en el Anexo I del R.D. distingan, entre condiciones diagnósticas, necesidad de la exploración neurológica sistemática, completa y extremadamente rigurosa, prerrequisitos, hallazgos cardinales, límites en la valoración de los datos, periodo de observación y pruebas instrumentales de soporte diagnóstico.

Los tres hallazgos cardinales en la muerte cerebral son: coma arreactivo, ausencia de reflejos troncoencefálicos $\mathrm{y}$ apnea. Los documentos recogen orientaciones prácticas muy válidas para la

38. Escudero Augusto, D. Diagnóstico clínico de muerte encefálica. Prerrequisitos y exploración neurológica.http://www.trasplan.org/Espanol/Muerte.htm.

39. Practice parameters for determinig brain death in adults (summary statement). Report of Quality Standars Subcommittee of American Academy of Neurology. Neurology, 1995; 45: 1012-1014.

40. Real Decreto 2070/1999, de 30 de diciembre, por el que se regulan las actividades de obtención y utilización de órganos humanos y la coordinación territorial en materia de donación y trasplante de órganos y tejidos.

41. Donación y trasplante de órganos. Valoración del donante potencial:diagnóstico de muerte cerebral. info_sanitaria/proceso/diagnostico.htm. http://donación.organos.ua.es/

42. Fernández G., Fernández F., Rey G, Concha T, Medina V, MenÉndez C Muerte encefálica y donación en población infantil. An Pediatr (Barc). 2004 May; 60(5): 450-453.

43. PÉrez-PÉrez RM, Bardalet-Vinals N, Soler-Murall N Diagnostico de muerte y trasplante de órganos: Implicaciones jurídicas y medicolegales. Med Clin (Barc) 2006 May 13; 126(18): 707-711.

44. Molina-Martínez FJ, TARogi_SÁnchez S, Barcelo-Artigues MI, Barcelo-Rossello A.Muerte encefálica y donación de órganos: Revisión a la luz de la legislación española. Rev Neurol. 2003 Apr 16-30; 36(8): 771-780.

45. Chamorro C, Romera MA, Silva Aturdimiento miocardico en la muerte encefálica. Med intensiva. 2006 Aug-Sep; 30(6): 299-300.

46. Recogido como Anexo I .al final del texto del R.D. 2070/1999. 
obtención de esos hallazgos, ya que (aunque no difíciles de realizar, cuando se tiene experiencia) necesitan una cuidadosa aplicación que evite interpretaciones erróneas de los mismos.

Las pruebas de confirmación diagnósti$c a$ han sido elementos de discusión durante muchos años ${ }^{47}$. Creo que se ha enfatizado demasiado en ello, haciendo creer que las pruebas de confirmación son "el criterio" para establecer la muerte cerebral. Quiero insistir, pues, en que la evidencia es resultado de un juicio clínico diagnóstico y no consiste en una prueba concreta, específica o selectiva, para poder hacer el diagnóstico de muerte cerebral. Ninguna prueba conocida puede, por sí sola, superar los niveles de evidencia que se alcanzan con el juicio clínico sintético de una correcta valoración neurológica.

\section{3. ¿Por quién es muerte cerebral?}

El diagnóstico clínico de muerte cerebral requiere que el que lo realiza posea la destreza y experiencia necesaria para un ponderado juicio de valor. La formación adecuada desde un punto de vista médico es esencial, así como la adecuada especialización y experiencia sobre problemas neurológicos, en los distintos procesos morbosos que pueden afectar al sistema nervioso central.

En general, esta labor es desempeñada, en la practica clínica habitual, por los neurólogos clínicos y los neurocirujanos, por lo que parece natural les corresponda la valoración diagnóstica, tal y como recoge la actual ley española ${ }^{48}$. Esta valoración legal es conveniente realizarla siguiendo un protocolo clínico, que debería ser elaborado por los servicios e instituciones, según una pauta que podría seguir los criterios diagnósticos y de exploración práctica sugeridos en el Anexo I del R.D. que ha desarrollado la aplicación de la Ley. En muchos hospitales existen protocolos que deben ser cumplimentados clínicamente en cada caso.

Una vez establecida la muerte cerebral clínica se plantea la necesidad de una fór- mula legal; a efectos de la certificación de la muerte el R.D. exige para la donación de órganos un certificado de defunción que permita la consideración y tratamiento como cadáver, y un certificado médico firmado por tres médicos, entre los que debe figurar un Neurólogo o Neurocirujano y el Jefe de Servicio de la Unidad médica donde se encuentre ingresado.

4. ¿Es definitivo el concepto actual de muerte cerebral?

El contexto cultural vigente, en el que es posible hacer diagnósticos de muerte cerebral, se encuentra potencialmente abierto a otros grados de evidencia más perfecta, es decir, a la perfectibilidad o progreso de la evidencia, con otros modos de entender el "constructo cultural", según lo permitan los futuros conocimientos científicos del momento.

\section{5. ¿Para qué es muerte cerebral?}

La muerte clínica es una consecuencia diagnóstica "desde el obrar" con un fin. Todo saber práctico o de lo concreto es un "saber para". La muerte clínica, en general es un diagnóstico que se hace "para algo", para conseguir algún fin.

Históricamente, el diagnóstico de muerte clínica lo han tenido que hacer los médicos probablemente con el único fin de evitar la putrefacción del cadáver ante los ojos. Es un sentir universal de cualquier cultura el horror al cadáver en putrefacción y especialmente si éste era un ser humano, y aún más si había sido conocido y querido por cuantos asistían a su muerte.

Esta ha sido la labor tradicional del médico ante la muerte: hacer un diagnóstico de muerte clínica "para enterrar".

El desarrollo de la medicina en nuestro siglo ha contribuido, sensiblemente, a modificar el panorama de los fines perseguidos para el diagnóstico de muerte. En efecto, dos nuevos fines aparecen con el desarrollo de las unidades de cuidados intensivos a partir de los años cincuenta. La muerte clínica, "para desconectar"; y la muerte clínica, "para trasplante".

47. Tema monográfico Muerte encefálica en UCI (II). Medicina intensiva 2000, 24: 143-198.

48. Ley 27-10-1979, núm. 30/1979, sobre extracción y trasplante de órganos. 


\section{6. ¿Cuándo es muerte cerebral?}

Solo existe muerte cuando hay muerte encefálica y la muerte cardiorrespiratoria, sin reanimación, no es más que el diagnóstico prematuro de muerte, anticipada a los cinco a diez minutos necesarios para que el encéfalo, sin circulación, pierda sus funciones de modo irreversible, por anoxia y lisis neuronal.

En consecuencia, existe "momento de la parada cardiaca y/o respiratoria”, pero realmente es problemático decidir cuando es el momento de la muerte verdadera; esto es, de la encefálica. La muerte cerebral ocurre evidentemente en un período de tiempo, es un proceso biológico de degradación estructural, que alcanza un nivel de pérdida de funciones con irreversibilidad, pero dada la complejidad estructural y funcional del encéfalo, no se puede establecer biológicamente un momento o instante de la muerte. Realmente, es un proceso biológico de desestructuración orgánica. Así, en realidad, el llamado "momento de la muerte cerebral" quedaría reducido, en su referencia temporal, al momento en el que el observador experto alcanza la evidencia sobre los criterios objetivos de la exploración.

7. ¿Qué más incluye un testimonio verdadero de muerte clínica?

Hasta aquí hemos abordado la muerte clínica desde un punto de vista fundamentalmente científico y biológico, partiendo del análisis racional de los hechos y atendiendo preferentemente a los fines y consecuencias del concepto "muerte cerebral", es decir, hemos tomado un punto de vista empírico, positivista y teleológico que nos sirve para aproximarnos, lo más correctamente posible, al diagnóstico y para la ayuda en la toma de decisiones. Sin embargo, la muerte clínica no se agota en esta perspectiva que es la que constituye el origen de su aplicación práctica. Solamente una ética puramente utilitarista podría quedar satisfecha con una aproximación tan parcial.

Desde mi punto de vista la fundamentación del contracto cultural "muerte clínica" y su equivalente neurológico "muerte encefálica" tiene su raíz epistemológica en el imperativo ético y legal que marca el evento de la muerte y el estado resultante de su consideración como cadáver. Como nos ha enseñado Zubiri, es el encéfalo como órgano de la formalización humana el que le permite a la individualidad humana alcanzar su mismidad y la suficiencia constitucional para poder seguir realizándose formalmente como persona ${ }^{49}$. Sería la pérdida de la suficiencia constitucional por desestructuración orgánica de dicho órgano esencial para la formalidad humana la que conduciría necesariamente al estado irreversible de la muerte biológica y a la imposibilidad de reactualización de su propia formalidad de realidad. Sin sentir intelectivo, o dicho de otra forma, sin intelección sentiente, no existe posibilidad de una unidad coherencial primaria que permita al organismo humano vivir o realizarse como individualidad. La respuesta orgánica a esta insuficiencia es el proceso irreversible de la muerte en forma de rápida putrefacción del organismo entero. Es un proceso biológico en el que no se pueden establecer instantes o referencias fijas con respecto al transcurso temporal, pero en el que no existe posibilidad de recuperar el relato argumental de la vida ya vivida. La vida humana de la persona concreta ha terminado definitivamente en el mundo en el que estaba dando de sí. Posiblemente sea así, porque, como refería más arriba, el argumento de una vida humana se ha plenificado ya.

49. Trueba J.L., Cerebro y persona. Reflexiones sobre la suficiencia constitucional, en J MASIÁ (ed) Ser humano, persona y dignidad. (Dilemas éticos de la medicina actual 19), Universidad Pontificia de Comillas y Editorial Desclé de Brouwer, 2005. 